\title{
Habilidad del cuidado, sobrecarga percibida y calidad de vida del cuidador de personas en diálisis
}

\author{
Claudia Patricia Cantillo-Medina, Alix Yaneth Perdomo-Romero, Claudia Andrea Ramírez-Perdomo \\ Departamento de Enfermería. Facultad de Salud. Universidad Surcolombiana. Neiva. Huila. Colombia
}

Como citar este artículo:

Cantillo-Medina CP, Perdomo-Romero AY, Ramírez-Perdomo CA. Habilidad del cuidado, sobrecarga percibida y calidad del vida del cuidador de personas en diálisis. Enferm Nefrol. 2021 Abr-Jun;24(2):184-93

\section{Resumen}

Introducción: La Insuficiencia Renal Crónica representa un problema de salud mundial, cuya complejidad y terapias de reemplazo renal generan en la persona deterioro funcional y alteración en la capacidad de cuidado, razón por la cual es importante contar con el apoyo de un cuidador.

Objetivo: Describir las características de los cuidadores de las personas en diálisis, y analizar la asociación entre la habilidad del cuidado, la sobrecarga percibida y la calidad vida de estos cuidadores.

Material y Método: Estudio descriptivo, corte transversal. Se utilizaron 4 instrumentos: Ficha de caracterización de la díada, Inventario de Habilidad de Cuidado, Instrumento Calidad de Vida y la Escala de sobrecarga de Zarit.

Resultados: Participaron 68 cuidadores, principalmente mujeres $(77,94 \%)$, con bajo nivel de habilidad de cuidado, afectación negativa en las dimensiones física, social y espiritual de la calidad de vida, la mayoría pre-

\section{Correspondencia:}

Claudia Patricia Cantillo Medina E-mail: claudiacantillol@hotmail.com sentó ausencia de sobrecarga. Se encontró asociación significativa, entre habilidad de valor con la calidad de vida y sobrecarga percibida con las dimensiones física y social $(p<0,05)$.

Conclusión: el cuidado a las personas en diálisis fue brindado por mujeres principalmente, es necesario generar estrategias para mejorar la habilidad del cuidado, el cual se ve afectado por el impacto en la calidad de vida y los niveles de sobrecarga experimentados por el cuidador.

PALABRAS CLAVE: insuficiencia renal crónica; diálisis; cuidador familiar; habilidad; sobrecarga del cuidador; calidad de vida.

\section{Ability to care, perceived overload and quality of life of the caregiver of dialysis patients}

\section{Abstract}

Introduction: Chronic kidney disease represents a global health problem, the complexity of the disease and renal replacement therapies generate a functional deterioration and alteration in the capacity for care in the person, which is why caregivers are essential. 
Objective: To describe the characteristics of the caregivers of dialysis people, and analyze the association between the ability to care, the perceived burden and the quality of life of these caregivers.

Material and Method: Descriptive cross-sectional study. Four instruments were used: Survey characterization care dyad family caregiver, Caring Ability Inventory, Quality of Life Instrument and Zarit Overload Scale.

Results: 68 caregivers participated, mainly women (77.94\%), with a low level of ability to care, negative impact on the physical, social and spiritual dimensions of quality of life, the majority presented an absence of overload. A significant association was found between the ability to care and quality of life, and perceived overload with the physical and social dimensions of quality of life $(p<0.05)$.

Conclusion: The care of dialysis people was developed mainly by women, it is necessary to generate strategies to improve care skills, which is affected by the impact on quality of life and levels of caregiver burden.

KEYWORDS: chronic kidney disease; dialysis; family caregiver; skill; caregiver burden; quality of life.

\section{Introducción}

Por su creciente prevalencia, incidencia y morbimortalidad, la Insuficiencia Renal Crónica (IRC) representa un problema de salud mundial, consecuencia de múltiples factores de riesgo como las Enfermedades Cardiovasculares (ECV), Diabetes Mellitus (DM), Hipertensión Arterial (HTA) y Obesidad, además de diversas condiciones presentes en el envejecimiento ${ }^{1}$, asociadas al estilo de vida de las personas ${ }^{2}$. Desde la situación sociosanitaria se define como una enfermedad catastrófica y de alto costo por las alteraciones psicofísicas, creciente coste para los sistemas de salud, años de vida perdidos tanto para la persona en diálisis como para su familia ${ }^{3}$.

El individuo con IRC avanza hasta llegar a la etapa terminal, requiriendo ingresar a terapia de reemplazo renal (TRR), diálisis peritoneal (DP), Hemodiálisis (HD) o trasplante renal (TR), para restablecer y mantener la estabilidad de su organismo, controlar los síntomas urémicos y prolongar su vida ${ }^{4}$. Las diferentes TRR generan en la persona múltiples necesidades y problemas, por los cuales puede necesitar de asistencia continua en el hogar ${ }^{4}$, para garantizar la efectividad, seguridad y mejores desenlaces en salud ${ }^{5}$.

La persona en diálisis y sus allegados sufren el embate de la enfermedad y su tratamiento como una connotación social, con importante impacto dentro de su cotidianidad $^{6}$, por la complejidad en el manejo y los tratamientos ${ }^{7}$. Deben cumplir varias recomendaciones del régimen terapéutico como dieta, control de la ingesta de líquidos, toma de medicamentos, actividad física, cuidado de los accesos para la diálisis, que convierten el cuidado en un reto para todos ${ }^{8}$; así mismo, el deterioro funcional puede producir perdida en la autonomía ${ }^{9}$ disminución de la capacidad de cuidado ${ }^{10}$, lo cual implicaría dependencia en mayor o menor medida ${ }^{11}$, afectando la calidad de vida ${ }^{12}$ de la persona enferma y su familia.

Para el individuo en tratamiento dialítico el apoyo de un cuidador es indispensable? ${ }^{9}$ una vez iniciada la dependencia funcional se generan acciones personales a partir de un contrato tácito de mutualidad y reciprocidad, generando y manteniendo un vínculo especial ${ }^{13}$; quien cuida es importante en el proceso de recuperación del enfermo, los dos deben afrontar una serie de condiciones sociales que sumadas a su estado emocional y al drama derivado de la enfermedad, los enfrenta a diversos obstáculos ${ }^{14}$.

Ante la sobrecarga de funciones propias de su rol familiar y social, la persona que desempeña la labor de cuidar y atender las necesidades del otro, requiere desarrollar habilidad para cuidar y prepararse para ejercer su labor en el hogar ${ }^{15}$, adaptándose a nuevas condiciones para mejorar la salud y el bienestar de aquel a quien cuida.

El cuidado se convierte en un batallar diario contra la enfermedad, donde se realizan tareas monótonas y repetitivas, con una sensación de falta de control, que finalmente agota las reservas psicofísicas del cuidador, produciendo sobrecarga y afectando la calidad de vida ${ }^{14}$.

Teniendo en cuenta la complejidad del proceso salud enfermedad de las personas en diálisis, el impacto de las TRR, la alta demanda de cuidados, el efecto del acto de cuidar en los cuidadores, la necesidad de garantizar atención segura y brindar mejores condiciones de cuidado en los hogares, se requiere ampliar el conocimiento en esta área, para mejorar la calidad de cuidados. Por lo descrito anteriormente, este estudio buscó describir las características de los cuidadores de las personas en diálisis, y analizar la asociación entre la habilidad del 
cuidado, la sobrecarga percibida y la calidad vida de estos cuidadores.

\section{Material y Método}

Estudio descriptivo, de corte transversal, para la selección de la muestra se empleó un muestreo por conveniencia. Participaron 68 cuidadores que cumplieron los siguientes criterios de inclusión: mayor de 18 años; cuidador de persona con IRC en diálisis por un período mayor a tres meses. Se excluyeron a los cuidadores que presentaban diagnóstico de alteraciones cognitivas registradas en la historia clínica. Los receptores de cuidado fueron 68 personas asistentes regulares a un programa de hemodiálisis y dialisis peritoneal, de quienes se recogieron las características sociodemográficas, grado de dependencia y función mental. La recolección de la información se realizó entre junio y septiembre de 2019.

Los participantes fueron contactados en el servicio de TRR, se les dio a conocer el objetivo de la investigación y previa firma del consentimiento informado se recolectó la información, actividad realizada por las investigadoras y los auxiliares de investigación.

Los instrumentos utilizados fueron:

a) Ficha de caracterización de la díada persona con Enfermedad Crónica no Transmisible ECNT- cuidador familiar conformado por 42 ítems y tres dimensiones: identificación de condiciones de la diada, perfil sociodemográfico, percepción de carga y de apoyo, medios de información y comunicación; cuenta con validez facial y de contenido para América Latina ${ }^{16}$.

b) Inventario de Habilidad de Cuidado de Nkongho ${ }^{17}$, constituido por 37 ítems, organizado en 3 sub-escalas asociadas con el entendimiento del propio ser y de otros: conocimiento, valor y paciencia; clasificadas en los rangos Bajo (203 o menos), Medio $(203,1-220,2)$ y Alto $(220,3$ o más), donde las puntuaciones más altas indican mayor habilidad para cuidar de manera general. Para el contexto Colombiano este instrumento es válido y confiable con Alfa de Cronbach de 0,89 y estabilidad de $r=0,80^{18}$.

c) Escala de sobrecarga de Zarit ${ }^{19}$, evalúa la presencia y nivel de sobrecarga, conformada por 22 preguntas tipo Likert con rango de respuesta de 0 a 4 (nunca, casi nunca, a veces, muchas veces, siempre). Para la sumatoria de las respuestas, un resultado menor de 46 indica no sobrecarga, de 46 a 56 sobrecarga leve, mayor de 56 sobrecarga intensa; con Alfa de Cronbach de 0,86 para el contexto colombiano.

d) Instrumento Calidad de Vida propuesto por Betty Ferrell de la versión en español, versión cuidador familiar, 02-2013-GCPC-UNC; organizado en 35 ítems, comprende cuatro subescalas: las dimensiones física (5 ítems, con un rango de puntuación de 5-20), psicológica (14 ítems con un rango de puntuación de 14-56), espiritual (7 ítems con un rango de puntuación de 7-28) y social (9 ítems con un rango de puntuación de 9-36). Los valores de puntuación en las dimensiones física $(<12,5)$, social $(<22,5)$ y espiritual $(<17,5)$ equivalen a una percepción positiva en cada dimensión; en la psicológica $(<40)$, indica percepción negativa. EI proceso de validez y confiabilidad del instrumento en el contexto colombiano reportó un índice de validez de contenido general (IVCG) de 0,91 , con una consistencia interna medida a través del Alfa de Cronbach de 0,86.

La investigación fue aprobada por el Comité de Ética y Bioética de la Universidad Surcolombiana, acta $\mathrm{N}^{\circ} 007$ de 2018, teniendo en cuenta los principios bioéticos: respeto a la dignidad humana, privacidad, libertad de expresión y sentimientos, confidencialidad y reciprocidad.

Para el proceso de análisis de datos se ha utilizado Microsoft Excel ${ }^{\circledR} 2019$ y el programa estadístico SPSS ${ }^{\circledR}$ versión 23. Las variables cualitativas se presentaron como frecuencias absolutas y relativas. Para las variables cuantitativas se utilizó media y desviación típica (DE) o mediana, rango intercuartílico (RIQ) y mínimo-máximo, según el resultado del contraste de normalidad realizado través de la prueba de Kolmogorov-Smirnov. Para la comparación de medianas entre los grupos se utilizó la prueba Kruskal Wallis. El análisis correlacional entre variables fue realizado a través del coeficiente de correlación de Rho Spearman. En todos los casos se tuvo en cuenta un nivel de significación estadística cuando el valor $p<0,05$.

\section{Resultados}

Los cuidadores eran principalmente mujeres, con un promedio de edad $49,4 \pm 13,5$ años; un nivel de escolaridad y sociodemográfico bajo, dedicados principalmente 
al hogar y estado civil casados. Eran cuidadores únicos, dedicados al cuidado por un período mayor a 37 meses y por más de 20 horas diarias (Tabla $\mathbf{1}$ ).

En relación a la calificación global de las habilidades de cuidado, la mayoría se clasificó en un nivel bajo. La edad media en los diferentes grupos fue de $50,5 \pm 13,1$ años en el grupo calificado como nivel bajo, $48,4 \pm 13,1$ años en el grupo medio y $47,2 \pm 15,2$ años en el grupo de nivel alto. En la Tabla 1 se pueden consultar las diferentes características de los cuidadores según el nivel de habilidad en el cuidado.

En cuanto a la sobrecarga percibida por los cuidadores, los participantes en el estudio percibieron ausencia de sobrecarga. Se encontró percepción negativa tanto en el bienestar físico, como en el social y en el espiritual, lo cual indica que existe afectación en estas dimensiones

Tabla 1. Características de los cuidadores de personas en diálisis y habilidad del cuidado $(n=68)$.

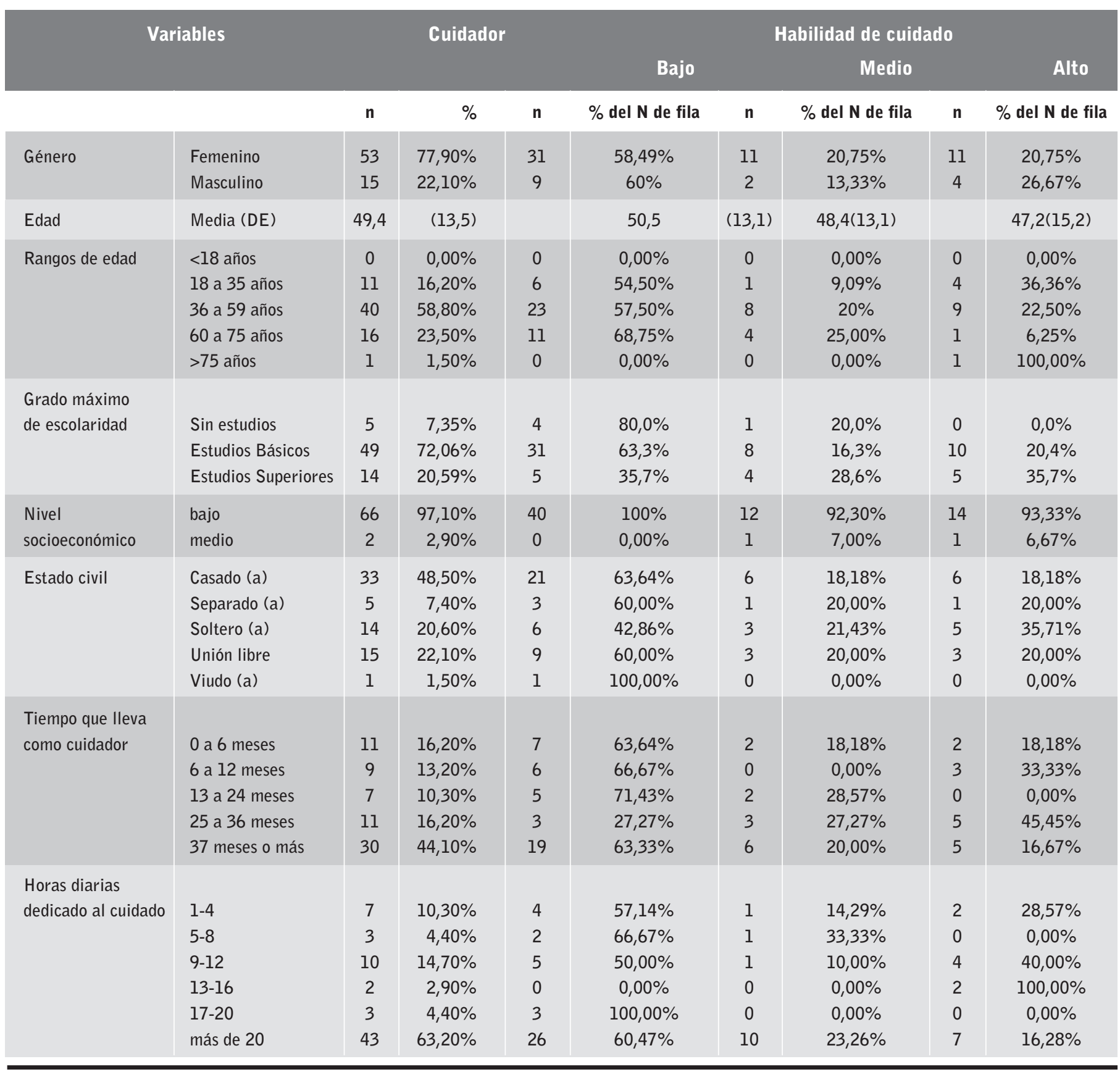


de la calidad de vida, mientras que en el bienestar psicológico las puntuaciones son congruentes con ausencia de afectación en esta dimensión (Tabla 2).

Al relacionar la sobrecarga percibida y la calidad de vida, se evidenció moderada relación estadísticamente significativa con el bienestar físico y con el bienestar social. El bienestar físico presentó relación estadísticamente significativa con el bienestar social y el bienestar psicológico con el bienestar espiritual. Además, presentaron relación estadísticamente significativa entre las categorías de habilidad de cuidado conocimiento, valor y paciencia (Tabla 3).

Se observaron relaciones significativas entre género con el bienestar espiritual; escolaridad con la dimensión social y nivel socioeconómico con bienestar físico. Adicionalmente relación estadísticamente significativa, entre tipo de TRR (Diálisis Peritoneal (DP) y Hemodiálisis (HD) de la persona con IRC y habilidad valor del cuidador, de igual manera relación estadísticamente significativa moderada de TRR (DP y HD) con el bienestar psicológico (Tabla 4).

Se evidenciaron relaciones estadísticamente significativas entre los tipos de TRR, DP (Diálisis Peritoneal Ambulatoria Continua (CAPD) y Diálisis Peritoneal Automatizada (APD)) de las personas con IRC, con habilidad valor de los cuidadores, bienestar social y bienestar psicológico (Tabla 4).

\section{Discusión}

La persona con IRC en diálisis afronta múltiples situaciones asociadas a la condición de cronicidad, circunstancia que requiere del soporte de un cuidador, recurso clave para la seguridad de la atención de salud. Los hallazgos obtenidos en este estudio permitieron describir la asociación de la habilidad del cuidado con la sobrecarga percibida y la calidad de vida del cuidador de personas dializadas, en una institución del sur de Colombia.

En cuanto a las características del cuidador valoradas se encontraron coincidencias con lo descrito en la literatura, el rol es asumido principalmente por mujeres ${ }^{7,14}$, 20-22, de 35 años de edad (media 49,4), en su etapa productiva laboralmente ${ }^{7,16}$; cuidan a personas con nivel medio de dependencia y función mental intacta ${ }^{10,23,24}$, bajo nivel educativo ${ }^{21,24-26}$ y socioeconómico, dedicados a labores del hogar21,24-28, estado civil casados, vínculo con la persona cuidada: esposos, e hijos ${ }^{29-31}$; tiempo dedicado al cuidado mayor a 37 meses y más de 20 horas diarias.

Lo referido anteriormente ubica al cuidador en condición de vulnerabilidad, debido al riesgo que enfrenta al asumir gran cantidad de roles ${ }^{7,16}$ en contextos poco favorables relacionados con aspectos educativos, económicos, sociales y emocionales, que afectan a las personas y familias al tener que modificar su estilo de vida, reajustar sus relaciones personales, laborales y sociales; cambios necesarios para afrontar los nuevos requerimientos de cuidar de la persona con insuficiencia renal ${ }^{6}$.

En referencia a las habilidades del cuidador, la mayoría puntuó en nivel bajo para la calificación global y en las categorías de conocimiento, paciencia y valor, similar a lo reportado por otros estudios ${ }^{20,25}$. Se encontró relación entre la habilidad de cuidado valor con el tipo de TRR (DP-HD), donde la experiencia de un pasado en el hogar y la sensibilidad a estar abierto a las necesidades del presente de la persona a cargo ${ }^{17}$, puede facilitar al cuidador brindar tratamiento y cuidado en casa, además de promover su adaptación a los nuevos y múltiples requerimientos de atención.

De igual manera se evidenció relación de la habilidad valor con el tipo de TRR DP (CAPD y APD) que puede ser generada al reconocer las fortalezas y debilidades propias de la diada ${ }^{17}$. Además, la asociación entre todas las categorías de habilidad de cuidado conocimiento (de sí mismo y su receptor de cuidado), paciencia (que permite encontrar un sentido al rol de cuidador) y valor (para tomar decisiones informadas y acceder a redes de apoyo social) ${ }^{17}$, las que sugieren existencia de factores que pueden ser modificados y se encuentran relacionados con la habilidad ${ }^{25}$, los cuales justifican ser abordados según las necesidades y requerimientos de los individuos, familias y sociedad.

Adicionalmente los resultados mostraron relación entre el tipo de TRR y el bienestar psicológico y el social de los cuidadores; razón por la cual se recomienda iniciar diálisis con terapias domiciliarias, pues en la medida que avanza la enfermedad se compromete la autonomía de la persona, haciéndose necesario continuar el tratamiento en los centros de diálisis; aspecto asociado a incapacidad laboral y unido a la dedicación de los cuidadores a labores no remuneradas en el hogar, contribuyen al empobrecimiento y vulnerabilidad de las familias 27,32 ; que al mismo tiempo genera estrés, 
Tabla 2. Calidad de vida y sobrecarga percibida en cuidadores de personas dializadas.

\begin{tabular}{|c|c|c|c|c|c|c|c|}
\hline Variable & & Mediana & Mínimo & Máximo & $\begin{array}{c}\text { Percentil } \\
25\end{array}$ & $\begin{array}{c}\text { Percentil } \\
75\end{array}$ & $\begin{array}{c}\text { Rango } \\
\text { intercuartil } \\
\text { (RIQ) }\end{array}$ \\
\hline Calidad de vida & $\begin{array}{l}\text { Bienestar Físico } \\
\text { Bienestar Psicológico } \\
\text { Bienestar Social } \\
\text { Bienestar Espiritual }\end{array}$ & $\begin{array}{l}12,0 \\
45,5 \\
22,0 \\
23,0\end{array}$ & $\begin{array}{c}5,0 \\
15,0 \\
10,0 \\
9,0\end{array}$ & $\begin{array}{l}20,0 \\
54,0 \\
32,0 \\
28,0\end{array}$ & $\begin{array}{c}9 \\
43 \\
19 \\
21\end{array}$ & $\begin{array}{l}15 \\
48 \\
25 \\
25\end{array}$ & $\begin{array}{l}6 \\
5 \\
7 \\
4\end{array}$ \\
\hline Sobrecarga del cuidador & & 28,0 & 8,0 & 63,0 & 40 & 62 & 21 \\
\hline
\end{tabular}

Tabla 3. Correlaciones Rho de Spearman entre habilidad de cuidado, sobrecarga y calidad de vida del cuidador $(n=68)$.

\begin{tabular}{|c|c|c|c|c|c|c|c|c|c|}
\hline & & Sobrecarga & $\begin{array}{l}\text { Bienestar } \\
\text { físico }\end{array}$ & $\begin{array}{c}\text { Bienestar } \\
\text { Psicológico }\end{array}$ & $\begin{array}{l}\text { Bienestar } \\
\text { social }\end{array}$ & $\begin{array}{l}\text { Bienestar } \\
\text { espiritual }\end{array}$ & Conocimiento & Valor & Paciencia \\
\hline Sobrecarga & $\begin{array}{l}\text { Coeficiente } \\
\text { de correlación } \\
\text { Spearman } \\
\text { Valor } p\end{array}$ & 1,000 & $\begin{array}{l}0,333 * * \\
0,006^{2}\end{array}$ & $\begin{array}{l}-0,122 \\
0,324\end{array}$ & $\begin{array}{c}0,541^{* * *} \\
0,000^{2}\end{array}$ & $\begin{array}{l}-0,195 \\
0,114\end{array}$ & $\begin{array}{l}-0,027 \\
0,828\end{array}$ & $\begin{array}{l}-0,128 \\
0,299\end{array}$ & $\begin{array}{l}-0,076 \\
0,540\end{array}$ \\
\hline $\begin{array}{l}\text { Bienestar } \\
\text { físico }\end{array}$ & $\begin{array}{l}\text { Coeficiente } \\
\text { de correlación } \\
\text { Spearman } \\
\text { Valor } p\end{array}$ & & 1,000 & $\begin{array}{l}0,142 \\
0,247\end{array}$ & $\begin{array}{l}0,301 * \\
0,0131\end{array}$ & $\begin{array}{l}0,034 \\
0,786\end{array}$ & $\begin{array}{l}0,094 \\
0,444\end{array}$ & $\begin{array}{l}0,116 \\
0,346\end{array}$ & $\begin{array}{l}0,096 \\
0,434\end{array}$ \\
\hline $\begin{array}{l}\text { Bienestar } \\
\text { psicológico }\end{array}$ & $\begin{array}{l}\text { Coeficiente } \\
\text { de correlación } \\
\text { Spearman } \\
\text { Valor } p\end{array}$ & & & 1,000 & $\begin{array}{l}0,216 \\
0,077\end{array}$ & $\begin{array}{l}0,317 * * \\
0,0082\end{array}$ & $\begin{array}{l}0,120 \\
0,331\end{array}$ & $\begin{array}{l}0,152 \\
0,217\end{array}$ & $\begin{array}{l}0,214 \\
0,080\end{array}$ \\
\hline $\begin{array}{l}\text { Bienestar } \\
\text { social }\end{array}$ & $\begin{array}{l}\text { Coeficiente } \\
\text { de correlación } \\
\text { Spearman } \\
\text { Valor } p\end{array}$ & & & & 1,000 & $\begin{array}{l}-0,056 \\
0,652\end{array}$ & $\begin{array}{l}-0,013 \\
0,916\end{array}$ & $\begin{array}{l}0,087 \\
0,479\end{array}$ & $\begin{array}{l}0,098 \\
0,425\end{array}$ \\
\hline $\begin{array}{l}\text { Bienestar } \\
\text { espiritual }\end{array}$ & $\begin{array}{l}\text { Coeficiente } \\
\text { de correlación } \\
\text { Spearman } \\
\text { Valor } p\end{array}$ & & & & & 1,000 & $\begin{array}{l}0,173 \\
0,158\end{array}$ & $\begin{array}{l}0,120 \\
0,329\end{array}$ & $\begin{array}{l}0,202 \\
0,098\end{array}$ \\
\hline Conocimiento & $\begin{array}{l}\text { Coeficiente } \\
\text { de correlación } \\
\text { Spearman } \\
\text { Valor } p\end{array}$ & & & & & & 1,000 & $\begin{array}{c}0,757 * * * \\
0,0002\end{array}$ & $\begin{array}{l}0,549 \\
0,0002\end{array}$ \\
\hline Valor & $\begin{array}{l}\text { Coeficiente } \\
\text { de correlación } \\
\text { Spearman } \\
\text { Valor } p\end{array}$ & & & & & & & 1,000 & $\begin{array}{c}0,566 * * * \\
0,000^{2}\end{array}$ \\
\hline Paciencia & $\begin{array}{l}\text { Coeficiente } \\
\text { de correlación } \\
\text { Spearman } \\
\text { Valor } p\end{array}$ & & & & & & & & 1,000 \\
\hline
\end{tabular}

*Correlación significativa baja; **Correlación significativa media; ***Correlación significativa alta; ${ }^{1}$ La correlación es significativa a nivel p $<0,05$ (bilateral); ${ }^{2}$ La correlación es significativa a nivel $p<0,01$ (bilateral). 
Tabla 4. Asociación entre características del cuidador de la persona en diálisis, habilidad de cuidado y calidad de vida del cuidador ( $n=68$ ).

\begin{tabular}{|c|c|c|c|c|c|c|c|c|c|c|c|c|c|c|}
\hline \multirow{3}{*}{ Variable } & \multicolumn{7}{|c|}{ Calidad de vida } & \multicolumn{7}{|c|}{ Habilidad de cuidado } \\
\hline & \multicolumn{2}{|c|}{$\begin{array}{l}\text { Bienestar } \\
\text { Físico }\end{array}$} & \multicolumn{2}{|c|}{$\begin{array}{c}\text { Bienestar } \\
\text { Psicológico }\end{array}$} & \multicolumn{2}{|c|}{$\begin{array}{l}\text { Bienestar } \\
\text { Social }\end{array}$} & \multicolumn{2}{|c|}{$\begin{array}{l}\text { Bienestar } \\
\text { Espiritual }\end{array}$} & \multicolumn{2}{|c|}{ Conocimiento } & \multicolumn{2}{|l|}{ Valor } & \multicolumn{2}{|c|}{ Paciencia } \\
\hline & Mediana & $\mathbf{p}$ & Mediana & $\mathbf{p}$ & Mediana & $\mathbf{P}$ & Mediana & $\mathbf{p}$ & Mediana & $\mathbf{p}$ & Mediana & $\mathbf{p}$ & Mediana & $p$ \\
\hline \multicolumn{15}{|c|}{ Sexo } \\
\hline $\begin{array}{l}\text { Hombre } \\
\text { Mujer }\end{array}$ & $\begin{array}{l}10 \\
12\end{array}$ & 0,05 & $\begin{array}{l}45 \\
46\end{array}$ & 0,749 & $\begin{array}{l}20 \\
22\end{array}$ & 0,256 & $\begin{array}{l}22 \\
24\end{array}$ & 0,045 & $\begin{array}{l}71 \\
69\end{array}$ & 0,474 & $\begin{array}{l}66 \\
66\end{array}$ & 0,942 & $\begin{array}{l}55 \\
56\end{array}$ & 0,612 \\
\hline \multicolumn{15}{|c|}{ Nivel Socioeconómico } \\
\hline $\begin{array}{l}\text { Bajo } \\
\text { Medio }\end{array}$ & $\begin{array}{l}12 \\
12\end{array}$ & 0,038 & $\begin{array}{l}45 \\
47\end{array}$ & 0,171 & $\begin{array}{l}22 \\
23\end{array}$ & 0,280 & $\begin{array}{l}23 \\
25\end{array}$ & 0,572 & $\begin{array}{l}67 \\
73\end{array}$ & 0,124 & $\begin{array}{l}66 \\
61\end{array}$ & 0,064 & $\begin{array}{l}55 \\
57\end{array}$ & 0,415 \\
\hline \multicolumn{15}{|c|}{ Escolaridad } \\
\hline $\begin{array}{l}\text { Sin Estudios } \\
\text { Estudios } \\
\text { Básicos } \\
\text { Estudios } \\
\text { Superiores }\end{array}$ & 12 & 0,469 & 47 & 0,480 & $\begin{array}{l}21 \\
21\end{array}$ & 0,023 & $\begin{array}{c}23 \\
23,5\end{array}$ & 0,060 & $\begin{array}{l}67 \\
78\end{array}$ & 0,353 & $\begin{array}{c}61 \\
74,5\end{array}$ & 0,110 & $\begin{array}{c}55 \\
59,5\end{array}$ & 0,797 \\
\hline \multicolumn{15}{|c|}{ Tipo de diálisis } \\
\hline $\begin{array}{l}\text { DP } \\
\text { HD }\end{array}$ & $\begin{array}{l}12 \\
12\end{array}$ & 0,749 & $\begin{array}{l}46 \\
44\end{array}$ & 0,003 & $\begin{array}{l}23 \\
21\end{array}$ & 0,653 & $\begin{array}{l}23 \\
23\end{array}$ & 0,887 & $\begin{array}{l}71 \\
67\end{array}$ & 0,353 & $\begin{array}{l}71 \\
58\end{array}$ & 0,014 & $\begin{array}{l}56 \\
55\end{array}$ & 0,697 \\
\hline \multicolumn{15}{|c|}{ Tipo de DP } \\
\hline $\begin{array}{l}\text { APD } \\
\text { CAPD }\end{array}$ & $\begin{array}{l}13 \\
11\end{array}$ & 0,548 & $\begin{array}{l}46 \\
47\end{array}$ & 0,014 & $\begin{array}{c}23,5 \\
18\end{array}$ & 0,008 & $\begin{array}{c}23 / 22,5 \\
23 / 25\end{array}$ & 0,066 & $\begin{array}{c}75,5 \\
61\end{array}$ & 0,784 & $\begin{array}{c}72,5 \\
47\end{array}$ & 0,009 & $\begin{array}{c}57,5 \\
52\end{array}$ & 0,852 \\
\hline
\end{tabular}

Prueba Kruskall Wallis

DP: Diálisis peritoneal; HD: Hemodiálisis; APD: Diálisis Peritoneal Automatizada; CAPD: Diálisis Peritoneal Ambulatoria Continua.

al estar obligado a asumir requerimientos no cubiertos por el sistema de salud ${ }^{25,28}$.

De igual forma se encontró asociación positiva entre el género con la dimensión espiritual, recurso que proporciona y puede aportar de manera positiva bienestar al cuidador y mejorar el proceso de adquisición de habilidades para cuidar ${ }^{21}$. Por otra parte, relación entre escolaridad y bienestar social, los altos niveles de escolarización garantizan mayor calidad en las funciones del $\mathrm{rol}^{21}$, aumento en la capacidad para cuidar de sí mismo $^{26}$ y, apoyo al cuidado seguro y continuo de los

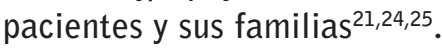

Asimismo, relación del nivel socioeconómico con bienestar físico, el cuidar a una persona con ECNT genera una nueva forma de afrontar las relaciones familiares, la asistencia directa sumada a actividades domésticas no remuneradas, ocasiona sentimientos de obligación del cuidador Ilevándolos a abandonar sus labores para involucrarse por completo con la persona cuidada ${ }^{29}$, 10 anteriormente descrito puede producir impacto económico, que terminaría por afectar el bienestar físico manifestado en el cansancio ${ }^{23}$, y con la percepción de sobrecarga asociada al cuidado.

Cabe agregar que existe relación entre las dimensiones del bienestar físico con el social y, psicológico con el espiritual. Al igual asociación positiva entre la sobrecarga experimentada y las dimensiones de bienestar físico y social; brindar cuidados informales en el hogar genera impacto negativo en el cuidador en el trabajo, en el hogar y en las relaciones interpersonales, además de crear una carga económica ${ }^{23}$; ellos experimentan desgaste físico y emocional por la sobrecarga de roles lo cual se agudiza por la falta de redes de apoyo social que contribuyan con estas tareas ${ }^{21}$.

Los hallazgos indican que existen variables del cuidador y de la persona en diálisis relacionadas con la ha- 
bilidad de cuidado, sobrecarga percibida y dimensiones de la calidad de vida; las cuales son factibles de intervenir e impactar en la labor de cuidado ${ }^{33}$. De acuerdo con los resultados encontrados se evidencia un nivel inadecuado de habilidad en los cuidadores, presencia de sobrecarga asociada a la demanda de cuidado, y manejo complejo de la enfermedad, con percepciones negativas en la mayoría de las dimensiones de la calidad de vida, circunstancias concordantes con lo referido en otros estudios $23,25,33$, aspectos importantes de ser abordadas con fortalecimiento continuo del vínculo entre la persona dializada, cuidador y equipo de salud.

Lo anteriormente descrito, es útil para mejorar las prácticas de cuidado, permite plantear intervenciones que incluyan al cuidador y a la persona en diálisis para empoderarlas de su cuidado, fortalecer la habilidad del cuidado, disminuir la sobrecarga e impactar la calidad de vida de los cuidadores, con adecuado entrenamiento, acompañamiento, seguimiento y evaluación continua, para garantizar la seguridad y mejores desenlaces en salud.

Como conclusión se puede afirmar que el cuidado a las personas en diálisis fue brindado por mujeres principalmente, quienes presentan baja habilidad del cuidado, el cual se observó afectado por el impacto en la calidad de vida y los niveles de sobrecarga experimentados. Se hace necesario generar estrategias para mejorar la habilidad e impactar de manera positiva la calidad de vida y minimizar el riesgo de sobrecarga.

\section{Agradecimientos}

Se agradece a la Universidad Surcolombiana por el apoyo recibido, cuidadores de personas con IRC dializadas por su participación en la investigación; así como al Grupo de Cuidado al Paciente Crónico de la Universidad Nacional de Colombia.

Los autores declaran no tener conflicto de interés.

Recepción: 04-10-20

Aceptación: 02-04-21

Publicación: 30-06-21

\section{Bibliografía}

1. KDOQI Clinical Practice Guideline for Hemodialysis Adequacy: 2015 Update. AJKD 2015; 66 (5):884-930.

2. Veras RP, Oliveira M. Aging in Brazil: the building of a healthcare model. Cien Saúde Colet. 2018; 23(6):1929-36.

3. Documento Marco sobre Enfermedad Renal Crónica (ERC) dentro de la Estrategia de Abordaje a la Cronicidad en el SNS. Ministerio de Sanidad Servicios Sociales e Igualdad. 2015.

4. Tejada Tayabas LM, Partida Ponce $K L$, Hernández Ibarra LE, María L. Coordinated hospital-home care for kidney patients on hemodialysis from the perspective of nursing personnel. Rev Latino-Am Enfermagem. 2015;23(2):225-33.

5. Daugirdas JT, Sherman RA, Ing TS. Complicaciones durante la hemodiálisis. Manual de diálisis. $5^{\text {ta }}$ edición. Philadelphia, PA. Wolters Kluwer; 2015.

6. Ramírez CA. Aprender a vivir con un órgano trasplantado. Rev. cienc. cuidad. 2019;16(3):93-102. doi: https://doi.org/10.22463/17949831.1596.

7. Alcaraz N, Vázquez JA, Pineda MT, Ramos FJ. La trayectoria de cuidado del paciente en hemodiálisis: de la noticia inesperada al desenlace final. Enferm Nefrol. 2019 Jul-Sep;22(3):308-16.

8. Cuevas-Budhart MA, Saucedo RP, Romero G, García JA, Hernández A. Relación entre las complicaciones y la calidad de vida del paciente en hemodiálisis. Enferm Nefrol 2017;20(2):112-9.

9. Álvarez M, Chocarro L, Velarde JF, Palacios D. La experiencia de ser portador de un catéter venoso central para hemodiálisis: Estudio cualitativo. Enferm Nefrol. 2018;21(2):146-54.

10. McQuoid J, Welsh J, Strazdins L, Griffin AL, BanweII C. Integrating paid work and chronic illness in daily life: A space-time approach to understanding the challenges. Heal Place. 2015;34:83-91.

11. Niu HY, Liu JF. The psychological trajectory from diagnosis to approaching end of life in patients undergoing hemodialysis in China: A qualitative study. Int J Nurs Sci 2017;4(1):29-33. 
12. Morais Arruda CG, Gomes Nascimento PMB, Medeiros SM, Raniere de Oliveira CR, Santos CM. Calidad de vida en pacientes con insuficiencia renal crónica en hemodiálisis. Enferm. glob. 2016;5(43):59-73.

13. Lazar A, Demiris G, Thompson HJ. Involving family members in the implementation and evaluation of technologies for dementia: a dyad case study. J. Gerontol. Nurs. 2015;41(4):21-6.

14. Ramírez CA, Salazar Y, Perdomo AY. Quality of life of caregivers of persons with neurological disorder sequels. Rev Cient la Soc Esp Enferm Neurol. 2017;45:9-16.

15. Carrillo GM, Barreto RV, Arboleda LB, Gutiérrez OA, Melo BG, Ortiz VT. Competencia para cuidar en el hogar de personas con enfermedad crónica y sus cuidadores en Colombia. Rev. Fac. Med. 2015; 63(4):668-75.

16. Chaparro L, Sánchez B, Carrillo G. Encuesta de caracterización del cuidado de la díada cuidador familiar - persona con enfermedad crónica. Rev Cien Cuidad. 2014;11(2):31-45.

17. Nkongho N. The Caring Ability Inventory. En: Strickland OL, Waltz CF. Measurement of Nursing Outcomes: Self Care and Coping. New York: Springer Publishing Company; 1996.

18. Barrera L, Sánchez B, Carrillo G, Chaparro L, Carreño $S$. Validación semántica y prueba de estabilidad del "Inventario de habilidad de cuidado" versión en español. Actualizaciones en Enfermería. 2015;18(1):8-14.

19. Barreto R, Campos M, Carrillo G, Coral R, Chaparro L, Durán et al. Entrevista Percepción de Carga del Cuidado de Zarit: pruebas psicométricas para Colombia. Aquichan. 2015;15(3):368-80.

20. Benítez M, Carreño S. Habilidad de cuidado y sobrecarga en cuidadores familiares de personas con cáncer. RFS. 2015;7(2):9-14.

21. Martínez L, Fernández E, González E, Vázquez HL. Apoyo social y resiliencia: factores protectores en cuidadores principales de pacientes en hemodiálisis. Enferm Nefrol. 2019 AbrJun;22(2):130-9.
22. Fernández Tijero $\mathrm{M}^{\mathrm{a}} \mathrm{C}$. El origen de la mujer cuidadora: apuntes para el análisis hermenéutico de los primeros testimonios. Index Enferm. 2016;25(12).

23. Romero E; Bohórquez C; Castro K. Calidad de vida y sobrecarga percibida por cuidadores familiares de pacientes con enfermedad renal crónica, Cartagena (Colombia) Archivos de Medicina. 2018;18(1):10513.

24. Aldana EA, Barrera SY, Rodríguez KA, Gómez OJ, Carrillo GM. Competencia para el cuidado (CUIDAR) en el hogar de personas con enfermedad renal crónica en hemodiálisis. Enferm Nefrol. 2016; 19(3):265-73.

25. Torres Pinto X, Carreño Moreno S, Chaparro Díaz L. Factores que influencian la habilidad y sobrecarga del cuidador familiar del enfermo crónico. Rev Univ Ind Santander Salud. 2017;49(2):3308.

26. Pérez C, Riquelme G, Scharager J, Armijo I. Relación entre calidad de vida y representación de enfermedad en personas con enfermedad renal crónica terminal en tratamiento con hemodiálisis. Enferm Nefrol. 2015;18(2):89-96.

27. Bona K, Dussel V, Orellana L, Kang T, Geyer R, Feudtner $C$ et al. Economic impact of advanced pediatric cancer on families. J Pain Symptom Manage. 2014;47(3):594-603.

28. Pereira LSM, Soares SM. Factors influencing the quality of life of family caregivers of the elderly with dementia. Cien Saúde Colet. 2015; 20(12):383951.

29. Hernández SM, Torres ML, Barrero SS, Saldaña IJ, Sotelo KD, Carrillo AJ, Rodríguez KA. Necesidades de cuidado paliativo en hemodiálisis percibidas por pacientes, cuidadores principales informales y profesionales de enfermería. Enferm. Nefrol 2019 AbrJun;22(2):141-9.

30. Blanco I, Leiva JP, Bolaños G, Jaume C, Julià JM, Rodríguez MA. Evaluación de necesidades paliativas en el paciente con enfermedad renal crónica en hemodiálisis. Enferm Nefrol. 2017;20(3):21520. 
31. Lau S, Lu X, Balsamo L, Devidas M, Winick N, Hunger SP et al. Family life events in the first year of acute lymphoblastic leukemia therapy: a children's oncology group report. Pediatr Blood Cancer. 2014; 61(12):2277-84.

32. Julián Mauro JC, Muñoz Carrasquilla S, Rosado Lázaro I. Factores asociados a la integración laboral de las personas en tratamiento renal sustitutivo en España. Enferm Nefrol. 2020;23(2):176-83.
33. Arroyo Priego E, Arana Ruiz AI, Garrido Blanco R, \& Crespo Montero R. Análisis de la sobrecarga del cuidador del paciente en diálisis. Enferm Nefrol. 2018;21(3):213-23.

Este artículo se distribuye bajo una Licencia Creative Commons Atribución-NoComercial 4.0 Internacional. https://creativecommons.org/licenses/by-nc/4.0/

\section{Open Access (c) (7) (8)}

